\title{
Different Electrical Properties of Paraspinal Muscle in Young Adults With Non-specific Chronic Low Back Pain and Healthy Individuals by Electrical Impedance Myography: A Cross-sectional Study
}

\section{Hongjiang Wang}

First Affiliated Hospital of Sun Yat-sen University

\section{Qiuhua Yu}

First Affiliated Hospital of Sun Yat-sen University

\section{Ziyan Fan}

First Affiliated Hospital of Sun Yat-sen University

Jiaxuan Zheng

First Affiliated Hospital of Sun Yat-sen University

Wai Leung Ambrose Lo

First Affiliated Hospital of Sun Yat-sen University

Le Li

Northwestern Polytechnical University

Chuhuai Wang ( $\nabla$ wangchuh@mail.sysu.edu.cn )

First Affiliated Hospital of Sun Yat-sen University

\section{Research Article}

Keywords: non-specific chronic low back pain, electrical impedance myography, rehabilitative ultrasound, lumbar paraspinal muscle

Posted Date: August 20th, 2021

DOI: https://doi.org/10.21203/rs.3.rs-818756/v1

License: (c) (i) This work is licensed under a Creative Commons Attribution 4.0 International License. Read Full License 


\section{Abstract}

\section{Background}

Non-specific chronic low back pain (NSCLBP) is a multifactor disorder with high prevalence rate of people all around the world. Lumbar paraspinal muscle (LPM) plays an important role of spine stabilization. However, the electrical properties of LPM in the patients were unclear. Aim to explore the electrical properties of bilateral LPM between patients with NSCLBP and healthy controls (HC), the study was designed to applied electrical impedance myography (EIM) technique to measure the LPM.

\section{Methods}

A total of thirty participates (15 in NSCLBP group, 15 in HC group) were instructed to have each side of LPM measured at the rest state and the maximum volunteer contraction (MVC) state. Then measured the EIM parameters of LPM at L4/5 level by a multi-frequency device. Each measurement repeated three times. The EIM parameters at $50 \mathrm{kHz}, 100 \mathrm{kHz}, 200 \mathrm{kHz}$ current frequencies were enrolled into the statistical analysis.

Results

At the three current frequencies, parameters in the right side of LPM had not significantly difference from the left ( $P>0.05$, respectively). Resistance (R) of LPM in NSCLBP group was larger than that in HC group $(P<0.05$, respectively), phase angle (PA) in NSCLBP group was smaller than that in $\mathrm{HC}$ group $(P<0.05$, respectively) and there was no difference of the reactance value between the two groups $(P>0.05$, respectively). At $50 \mathrm{kHz}$, relationships between the EIM parameters and geometric parameters of LPM were not significant in NSCLBP group or $\mathrm{HC}$ group ( $P>0.05$, respectively).

\section{Conclusion}

The electrical properties of bilateral LPM in young adults with NSCLBP were different from those of healthy individuals regardless of any current frequency. The ultrasound parameters of LPM, however, showed no significant difference between young patients with NSCLBP and healthy participants. EIM measurement might be more sensitive than rehabilitative ultrasound for NSCLBP in young adults.

Trial registration

The Chinese Clinical trial registration number: ChiCTR2100043113.

\section{Introduction}

Chronic low back pain (CLBP) is a multifactor disease all around the world, its lifetime prevalence is 75$84 \%$ in developed countries[1]. It highly occurs on every grade of years-old level[2]. $80 \%-90 \%$ of patients with CLBP are non-specific (NSCLBP), intractable, and difficult to cure[3]. A lot of researchers argued that 
the pathogenesis of NSCLBP remains controversial[4]. Since Panjabi firstly suggested the stabilizing system of spine: instability hypothesis of low back pain[5], people focus on studying the function of trunk muscle of the patients. Lumbar paraspinal muscle (LPM) such as erector spinae muscle, lumbar multifidus and transversus abdominis were found to be important for core stabilization[6].

Electrical impedance myography (EIM) can be a new technique for measuring the electrical properties of LPM measurement of patients with NSCLBP. EIM is an evolution of bioelectrical impedance analysis relies upon the application and measurement of high-frequency, low-intensity electrical current, which is widely used as a noninvasive method for body composition assessment of neuromuscular diseases[7], includes body myositis[8], Duchenne muscular dystrophy[9], amyotrophic lateral sclerosis[10], subacute stroke[11], facioscapulohumeral muscular dystrophy[12] and so on. EIM is reliability, highly reproducible and easier to operate[13,14]. Different from conventional needle electromyography and most standard neurophysiological techniques, EIM does not focus on measuring the inherent electrical activity of the tissues. Rather, its measurements are made over a small area of interest, with energy being applied to the body and the resultant surface patterns analyzed. Unlike ultrasound, however, in which energy is in the form of sound waves and the main interest is image reconstruction, in the case of EIM, electrical current is used and the output is a set of quantitative parameters describing the state of the muscle, with presently little emphasis on imaging[7]. In the study of acute LBP[15], it was found the intracellular resistance in patients was higher than healthy controls. However, it had not the reactance and phase angle and the correlation between electrical properties and thickness of muscle into the analysis. It offered the possibility of the electrical properties of LPM measurement. By now, there is little knowledge about the resistance, reactance of LPM in NSCLBP patients as patients with NSCLBP were different from that with acute LBP[16].

Whether the electrical properties of LPM in NSCLBP patients were different from healthy individuals and if it was correlated to the thickness or contraction of LPM very interested us. For patients with NSCLBP, we gave a hypothesis that the resistance, reactance or phase angle would be different from healthy controls and the difference could be correlated to the morphological parameters of the muscle.

Aim to verify the hypothesis, we designed the study to compare the electrical impedance parameters and thicknesses of bilateral LPM at L4/5 level between individuals with NSCLBP and healthy controls (HC) in young adults.

\section{Methods}

It is a cross-sectional study to compare the electric properties and the morphological parameters of LPM among the subjects with NSCLBP and without. For further to explore the electrical conductivity of the muscle fibers, we detected the electrical properties of bilateral LPM at three different current frequencies, $50 \mathrm{kHz}, 100 \mathrm{kHz}$ and $200 \mathrm{kHz}$.

\section{Participants}


A total of thirty individuals (NSCLBP group, 15; HC group, 15) were recruited to participate in this study through advertisements on social platform and outpatient service. Inclusion criteria for the NSCLBP participants were as follow: medical diagnosis of non-specific LBP with pain and symptoms persisting for more than 3 months and visual analogue scale (VAS) $>3 \mathrm{~cm}$, for which medical treatment had been sought; aged 18-40 years; and without history of other diseases. Participants were excluded if they had any of the following: aged outside the 18-40 years range, LBP of traumatic or structural origin, LBP with neurological symptoms or pain radiation in the lower leg(s), previous back surgery, spinal tumors or infections, or neurological and/or musculoskeletal disorders unrelated to LBP [17].

For the $\mathrm{HC}$ group, the inclusion criteria was participants without low back pain in 2 years and had no history with diseases were included[18]. Meanwhile, those out of the age range, pregnant, and had history with other diseases or surgery were excluded from the story. The diagnosis of NSCLBP was referenced to the diagnostic guidelines published by the American College of Physicians and the American Pain Society[19].

\section{Procedure and data collection}

\section{Questionnaires}

Patients with NSCLBP were instructed to quantify the intensity of the pain were by Visual Analogue Scale (VAS), marked on a $10-\mathrm{cm}$ scale ruler from 0 to 10 (0 means no pain, 10 means severe pain). And filled an Oswestry Disability Index (ODI)[20] and a Roland-Morris Disability Questionnaire (RDQ)[21] to assess their pain-related disability and disfunction of daily life. Healthy controls who passed the screening were not application of these questionnaires (Details in table 1).

\section{Morphological parameters collection}

A rehabilitative ultrasound (SONIMAGE HS1, KONICA MINOLTA Inc., Japan) with a C5-2 curvilinear transducer was applied to measure the thickness of bilateral LPM muscles at L4/L5 level[22], participants were in prone, with a pillow under the abdomen to have the lumbosacral junction angle to less than $10^{\circ}$. The images of three-times repeated measurements at rest and maximum volunteer contraction (MVC) were acquired. When measuring the thickness at MVC, participates should lift the contralateral arm off the bed and holding it in $120^{\circ}$ of shoulder abduction and $90^{\circ}$ of elbow flexion[23]. The participates were instructed to familiar contracting before examination. The change percentage of muscles was calculated follow the formula[24]: Change $\%=\left(T_{M V C}-T_{\text {rest }}\right) / T_{\text {rest }} * 100 \%, T_{\text {rest }}$ means unilateral LPM thickness at rest, $\mathrm{T}_{\mathrm{MVC}}$ means unilateral LPM thickness at MVC. The operator of ultrasound examination was a professionally trained therapist who was blinded to subjects belongs to groups.

\section{EIM data collection and processing}

A multi frequency body composition analysis system (Imp SFB7, Inc., Sydney, NSW, Australia) was applied to measure the electrical impedance of LPM. ImpediMed SFB7 is a medical multifrequency 
device, capable of applying current simultaneously at a multitude of frequencies from $2 \mathrm{kHz}$ to $1 \mathrm{MHz}$. Impedance can be described mathematically. EIM parameters composed of its resistance $(R)$ and its two forms of reactance (capacitive reactance $\left(X_{C}\right)$ and inductive reactance $\left(X_{L}\right)$ ) in combination[7]. In medical applications it can be simplified by ignoring the $X_{L}$ term, since inductance is believed to play a minimal role in standard bioimpedance measurements, and the $\mathrm{X}_{C}$ term can be simply written as $\mathrm{X}$. The phase angle (PA) via standard trigonometric relationships by the formula[7] $\mathbb{P A}=\arctan (X / R)$. Two trained rehabilitative clinicians were involved in data collection who were unknown of which group the participates belonged to.

Participates were instructed in the prone position with a soft pillow placed on the front of the abdomen so that the lumbar arch kept straightness, then made the low back fully exposed. Before testing, the lumbar skin should be cleaned by $75 \%$ alcohol and wait until dry thoroughly. Each side of LPM, the upper edge of the third electrode was taken as the connecting level of $2 \mathrm{~cm}$ beside the spinal process and anterior superior iliac crest. At a longitudinal direction, arrange vertically along the ridge process and place other electrodes, the 1st, $2 \mathrm{nd}, 3 \mathrm{rd}$, and 4th electrodes (1 $\mathrm{st}$, 4th are current electrodes and $2 \mathrm{nd}$, 3rd are voltage electrodes) are separated by $20 \mathrm{~mm}, 30 \mathrm{~mm}$ and $20 \mathrm{~mm}$, respectively. (Fig 1a) Recorded PA, R and X value at $50 \mathrm{kHz}, 100 \mathrm{kHz}$, and $200 \mathrm{kHz}$. The contralateral LPM was detected by the same method (Fig $1 \mathrm{~b}$ ). The model of electrode plates was L-DEX (Lymphedema Dual Tab Electrode, Pinkenba QLD 4008, Australia). All the EIM data were calculated by the Analysis System, Version 5.5.0.1. To avoid the possible bias, an average value of the three measurements was in the statistical analysis[15]. The operators were two physiotherapists with more than three-years clinical experience and they were blind to which group the participates belonged.

\section{Statistical analysis}

Statistical analysis of parameters was performed using SPSS version 26.0 (IBM Statistics, New York, NY, USA). The Kolmogorov-Smirnov test was used to determine the normality of the data. Independent samples t-tests were used to compare the two groups for anthropometric measurements if they were normally distributed, while chi-square test was used to compare in terms of sex. If there was a significant interaction between the baseline factor, it would be regarded as a covariate in the ANOVA. General Liner Model (GLM) with multivariate analysis of covariance was used to compare the $R, X$ and $P A$ in the two groups within the bilateral LPM at the different current frequencies. Paired t-tests were applied to evaluate the differences between the left and right sides in each group. Spearman's rank $r$ was used to assess the correlation between the EIM parameters and the morphological parameters. A two-tailed $\mathrm{P}<0.05$ was considered statistically significant.

\section{Results}

\section{Characteristics of subjects in the NSCLBP group and in the HC group}


Thirty subjects were included in the study, fifteen in the NSCLBP group, fifteen in the HC group. There was no statistical difference at sex, height, weight and $\mathrm{BMI}$ in groups $(P=0.247, P=0.979, P=0.905, P=0.805$, respectively). However, the average age was different between NSCLBP group and HC group $(P=0.002)$ (Table 1).

Table 1 Demographics of subjects in NSCLBP group and HC group (Mean \pm SD)

\begin{tabular}{llll}
\hline Characteristic & NSCLBP & HC & $P$ \\
\hline Sex (Male/Female) & $3 / 12$ & $6 / 9$ & 0.247 \\
\hline Age (years) & $26.53 \pm 2.70$ & $23.20 \pm 2.76$ & $0.002^{\star}$ \\
\hline Height (cm) & $164.80 \pm 6.34$ & $164.73 \pm 7.30$ & 0.979 \\
\hline Weight (kg) ${ }^{\&}$ & $57.10 \pm 8.88$ & $56.67 \pm 10.69$ & 0.905 \\
\hline BMI\& & $20.94 \pm 2.27$ & $20.72 \pm 2.48$ & 0.805 \\
\hline VAS (cm) & $5.19 \pm 1.11$ & NA & \\
\hline ODI (points) & $8.00 \pm 4.33$ & NA & \\
\hline RDQ (pionts) & $3.53 \pm 2.53$ & NA & \\
\hline BMI, body & & & \\
\hline
\end{tabular}

$\mathrm{BMI}$, body mass index; VAS, visual analogue scale; ODI, Oswestry Disability Index; RDQ, Roland-Morris Disability Questionnaire. \# means chi-square test, \& means independent samples t-tests. NA means not application; $P$ value marked with * means significant difference compared to the control group $P<0.05$.

\section{Thickness and change percentage at each side of LPM in the two groups}

Compared to the HC group at the rest state, the thickness of unilateral LPM in the NSCLBP group showed no significant difference (Left $P=0.692$, right $P=0.431$ ) and no significant difference at the MCV state (Left $P=0.286$, right $P=0.314$ ). Both sides of LPM change percentage also showed no statistical difference between the two groups (Left $P=0.202$, right $P=0.893$ ) (Table 2). Paired t-test was applied to explore the difference between the left side and the right side of LPM, it was found no statistical significance $(P>0.05)$. 
Table 2 Thickness and change percentage of unilateral LPM (Mean \pm SD)

\begin{tabular}{lllll}
\hline Side & Group & $\mathrm{T}_{\text {rest }}(\mathrm{mm})$ & $\mathrm{T}_{\mathrm{MVC}}(\mathrm{mm})$ & Change\% (\%) \\
\hline Left & NSCLBP & $21.20 \pm 4.40$ & $28.65 \pm 5.48$ & $36.28 \pm 10.43$ \\
\hline & HC & $21.81 \pm 3.94$ & $30.71 \pm 4.89$ & $42.18 \pm 14.02$ \\
\hline \multirow{2}{*}{ Right } & $P$ & 0.692 & 0.286 & 0.202 \\
\hline & NSCLBP & $21.13 \pm 4.83$ & $29.68 \pm 5.92$ & $42.04 \pm 12.84$ \\
\hline & HC & $22.49 \pm 4.49$ & $31.75 \pm 5.08$ & $42.68 \pm 12.68$ \\
\hline
\end{tabular}

Independent t-test was applied. LPM means lumbar paraspinal muscle, $T_{\text {rest }}$ means unilateral LPM thickness at rest, $T_{M V C}$ means unilateral LPM thickness at MVC, Change\% means change percentage of unilateral LPM from the rest state to MCV state. $P<0.05$ means there is statistical significance.

\section{EIM parameters in the NSCLBP group and in the HC group}

As the age of the subjects in NSCLBP group and HC group was different, it was regarded as a covariate factor in the ANOVA to analysis the EIM parameters. The resistance $(R)$ values of the right LPM shew larger in NSCLBP group than in $\mathrm{HC}$ group at the current frequencies of $50 \mathrm{kHz}(P=0.019), 100 \mathrm{kHz}$ $(P=0.017), 200 \mathrm{kHz}(P=0.016)$, so as the left side at $50 \mathrm{kHz}(P=0.047), 100 \mathrm{kHz}(P=0.043), 200 \mathrm{kHz}$ $(P=0.044)$. The average phase angle (PA) values of the bilateral LPM were smaller in NSCLBP group than in $\mathrm{HC}$ group at $50 \mathrm{kHz}$ (left $P=0.003$, right $P=0.007$ ), $100 \mathrm{kHz}$ (left $P=0.019$, right $P=0.015$ ), $200 \mathrm{kHz}$ (left $P<0.001$, right $P=0.024)$. Details were illustrated in the Table 3 . 


\begin{tabular}{|c|c|c|c|c|}
\hline EIM parameter & Group & $50 \mathrm{kHZ}$ & $100 \mathrm{kHZ}$ & $200 \mathrm{kHZ}$ \\
\hline \multirow[t]{6}{*}{$\mathrm{R}$ (ohms) } & NSCLBP-left LPM & $32.11 \pm 7.29$ & $29.91 \pm 7.97$ & $28.15 \pm 7.22$ \\
\hline & HC-left LPM & $26.62 \pm 4.35$ & $24.28 \pm 4.47$ & $22.46 \pm 4.55$ \\
\hline & $P$ & $0.019^{*}$ & $0.017^{*}$ & $0.016^{*}$ \\
\hline & NSCLBP-right LPM & $33.25 \pm 9.03$ & $31.03 \pm 9.06$ & $29.29 \pm 9.05$ \\
\hline & HC-right LPM & $26.34 \pm 4.94$ & $24.01 \pm 4.99$ & $22.24 \pm 5.05$ \\
\hline & $P$ & $0.047^{*}$ & $0.043^{*}$ & $0.044^{\star}$ \\
\hline \multirow[t]{6}{*}{ X (ohms) } & NSCLBP-left LPM & $4.21 \pm 0.58$ & $3.90 \pm 0.53$ & $3.14 \pm 0.43$ \\
\hline & HC-left LPM & $4.49 \pm 0.58$ & $4.07 \pm 0.45$ & $3.17 \pm 0.39$ \\
\hline & $P$ & 0.089 & 0.336 & 0.923 \\
\hline & NSCLBP-right LPM & $4.26 \pm 0.72$ & $3.91 \pm 0.64$ & $3.12 \pm 0.49$ \\
\hline & HC-right LPM & $4.48 \pm 0.44$ & $4.03 \pm 0.34$ & $3.13 \pm 0.25$ \\
\hline & $P$ & 0.163 & 0.447 & 0.978 \\
\hline \multirow[t]{6}{*}{ PA (degrees) } & NSCLBP-left LPM & $7.77 \pm 1.81$ & $7.78 \pm 1.90$ & $6.70 \pm 1.66$ \\
\hline & HC-left LPM & $9.86 \pm 2.45$ & $9.89 \pm 2.62$ & $8.41 \pm 2.41$ \\
\hline & $P$ & $0.003^{* *}$ & $0.019^{*}$ & $<0.001^{\star \star}$ \\
\hline & NSCLBP-right LPM & $7.72 \pm 2.15$ & $7.67 \pm 2.28$ & $6.54 \pm 1.94$ \\
\hline & HC-right LPM & $9.99 \pm 2.32$ & $9.95 \pm 2.50$ & $8.44 \pm 2.27$ \\
\hline & $P$ & $0.007^{\star *}$ & $0.015^{\star}$ & $0.024^{*}$ \\
\hline
\end{tabular}

\section{Correlations between the electrical properties and morphological parameters of LPM in the NSCLBP group at $50 \mathrm{kHz}$}

Correlated analysis in the NSCLBP group shewed insignificant results at $50 \mathrm{kHz}$ current frequency. Little relationships were found between $P A, R, X$, thickness at rest and at MVC and change percentage of each side of LPM in the NSCLBP group $(P<0.05)$. And no significant correlation occurred in the HC group $(P<0.05)$ (Table 4). 
Table 4 Spearman correlation between the electrical properties and morphological parameters of LPM at $50 \mathrm{kHz}$

Group Left-PA Left-R Left-X Right-PA Right-R Right-X

\section{NSCLBP group}

\begin{tabular}{|c|c|c|c|c|c|c|c|}
\hline Left- $T_{\text {rest }}$ & $r$ & -0.200 & 0.150 & -0.300 & -0.271 & 0.036 & -0.457 \\
\hline & $P$ & 0.475 & 0.594 & 0.277 & 0.328 & 0.899 & 0.087 \\
\hline \multirow[t]{2}{*}{ Left-T ${ }_{M V C}$} & $r$ & -0.165 & 0.129 & -0.323 & -0.186 & 0.004 & -0.409 \\
\hline & $P$ & 0.557 & 0.647 & 0.241 & 0.506 & 0.990 & 0.130 \\
\hline \multirow[t]{2}{*}{ Left-Change\% } & $r$ & 0.114 & 0.171 & 0.389 & 0.132 & 0.121 & 0.314 \\
\hline & $P$ & 0.685 & 0.541 & 0.152 & 0.639 & 0.666 & 0.254 \\
\hline \multirow[t]{2}{*}{ Right- $T_{\text {rest }}$} & $r$ & -0.146 & 0.061 & -0.346 & -0.136 & -0.100 & -0.396 \\
\hline & $P$ & 0.603 & 0.830 & 0.206 & 0.630 & 0.723 & 0.143 \\
\hline \multirow[t]{2}{*}{ Right- $\mathrm{T}_{\mathrm{MVC}}$} & $r$ & -0.161 & 0.054 & -0.339 & -0.121 & -0.100 & -0.396 \\
\hline & $P$ & 0.567 & 0.850 & 0.216 & 0.666 & 0.723 & 0.143 \\
\hline \multirow[t]{2}{*}{ Right-Change\% } & $r$ & 0.029 & 0.143 & 0.457 & 0.021 & 0.182 & 0.200 \\
\hline & $P$ & 0.919 & 0.612 & 0.087 & 0.940 & 0.516 & 0.475 \\
\hline
\end{tabular}

HC group

\begin{tabular}{|c|c|c|c|c|c|c|c|}
\hline Left- $T_{\text {rest }}$ & $r$ & 0.089 & -0.289 & -0.100 & 0.029 & -0.207 & 0.039 \\
\hline & $P$ & 0.752 & 0.296 & 0.723 & 0.919 & 0.459 & 0.889 \\
\hline \multirow[t]{2}{*}{ Left-T MVC } & $r$ & 0.171 & -0.343 & 0.075 & 0.118 & -0.275 & 0.136 \\
\hline & $P$ & 0.541 & 0.211 & 0.791 & 0.676 & 0.321 & 0.630 \\
\hline \multirow[t]{2}{*}{ Left-Change\% } & $r$ & 0.054 & 0.104 & 0.300 & 0.064 & 0.054 & 0.093 \\
\hline & $P$ & 0.850 & 0.713 & 0.277 & 0.820 & 0.850 & 0.742 \\
\hline \multirow[t]{2}{*}{ Right- $T_{\text {rest }}$} & $r$ & 0.000 & -0.046 & 0.079 & -0.025 & 0.025 & 0.279 \\
\hline & $P$ & 1.000 & 0.869 & 0.781 & 0.930 & 0.930 & 0.315 \\
\hline \multirow[t]{2}{*}{ Right- $T_{M V C}$} & $r$ & 0.129 & -0.171 & 0.189 & 0.082 & -0.182 & 0.132 \\
\hline & $P$ & 0.648 & 0.541 & 0.499 & 0.771 & 0.516 & 0.639 \\
\hline Right-Change\% & $r$ & 0.275 & -0.289 & 0.275 & 0.300 & -0.439 & -0.157 \\
\hline
\end{tabular}




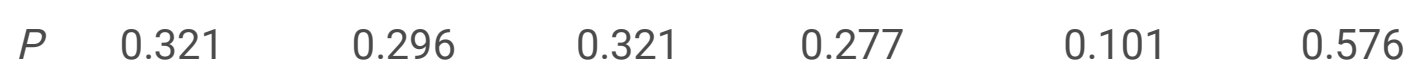

$-T_{\text {rest }}$ means the thickness of unilateral LPM at rest, $-T_{M V C}$ means the thickness of unilateral LPM at maximum volunteer contraction; r: correlation coefficient, -Change\% means the change percentage of unilateral LPM from rest to MCV. It was significant at $p<0.05$ with double tails.

\section{Discussion}

It is an initial objective of the present study was to identify the difference in EIM values of lumbar paraspinal muscle between non-specific NSCLBP patients and healthy individuals. The results showed the resistance was increased, the reactance was no change, the phase angle was decreased in patients with NSCLBP. No significant difference was found on the morphological parameters between the patients and healthy controls.

\section{Resistance and phase angle of LPM changed in patients with NSCLBP}

In muscle measured between the $\mathrm{kHz}$ and $\mathrm{MHz}$ range, the reactive component of cells is caused mainly by the myofiber membrane, which behaves as an electrical insulator[25]. The results showed the resistance value in the NSCLBP group increased, the phase angle decreased compared to HC groups and reactance value was indifferent. These findings are partly consistent with that of Ching et al[15] who assessed the electrical properties of LPM of patients with acute LBP, which reported the intracellular $\mathrm{R}$ value was larger than healthy subjects at high frequency current.

As the frequency is varied, the impedance characteristics are related to the underlying structure and properties of muscle. The impedance frequency dependence can be modeled empirically by Cole[25]. Shiffman et al[26] discussed extensions of multi-frequency EIM to $2 \mathrm{MHz}$ and exhibits new features which are found a major rise in the reactance of muscle above $3-500 \mathrm{kHz}$. It revealed major qualitative differences in the frequency dependences of $\mathrm{R}$ and $\mathrm{X}$ in healthy versus diseased states.

At $50 \mathrm{kHz}, 100 \mathrm{kHz}$ and $200 \mathrm{kHz}$, the differential trend between the groups was similar, the $\mathrm{R}$ value of bilateral LPM in NSCLBP groups were both larger than in HC group and the $\mathrm{X}$ value were indifferent at these three frequencies. Since the membrane permeability effector at low frequencies, only highfrequency current can flow through the intracellular compartment. The intracellular and extracellular matrices of muscle tissue act as resistors, and any atrophy which reduces the cross-sectional area of muscle tissue would increase the $\mathrm{R}$ value[27]. Changes in $\mathrm{X}$ value are related to changes in features of the cell membrane and layers of tissue[28]. Coutinho et al[29] suggested changes in reactance were likely brought about by changes in the features of the cellular membranes.

To reduce the impact of muscle anatomy on impedance analysis, PA was used to describe the relation between resistance and reactance[30], which evaluates membrane oscillation properties of the muscle. Consist with Wang et al[14], in the present study, the PA values were decreased in the NSCLBP group, which means the membrane oscillation properties of LPM changed in the patients with NSCLBP. Li et al[31] suggested the observations of PA decreased can be demonstrated two representative patterns of 
resistance and reactance relation. The first pattern shows larger resistance in the patient muscle and similar reactance values in the patient and healthy muscles across multiple frequencies. In the second pattern, the reactance was smaller in the patient muscle whereas resistance remained similar in both. In the current study, the results were belonged to the first pattern. It indicates the LPM in young adults with NSCLBP might have early atrophic changes with the cellular membrane oscillation properties decreased but the cellular membranes were intact.

\section{Thickness and change percentage of LPM showed no difference in the two groups}

In the study, the thickness and contraction function of bilateral LPM had not changed through comparing NSCLBP group with HC group. It is consistent with Sweeney et al[22] that they reported there were no differences between the groups for LM thickness or percentage thickness change in prone or standing at rest, or during activation with MVC in prone. However, other studies supported that the pattern of multifidus muscle atrophy in CLBP patients is localized rather than generalized but also provided evidence of a corresponding reduced ability to voluntarily contract the atrophied muscle[32]. Zhang et al[33] used ultrasound assessment to suggest the thickness, cross-sectional area (CSA) of lumbar multifidus was smaller in NSCLBP patients than healthy individuals. The patients with NSCLBP enrolled in the study were young adults which could be different from the older patients. This discrepancy may rely on the heterogeneity of non-specific CLBP[34] or aged-related factors[35].

\section{The EIM parameters and morphological parameters of LPM were resulted different in NSCLBP group compared to the healthy individuals}

The choice of using the $50 \mathrm{kHz}$ data was based on the fact that values obtained at this single frequency are most physiologically important as sensitive indicators of muscle status. The sensitivity stems from the fact reactance and phase values of healthy muscle peak at approximately $50 \mathrm{kHz}$, and in disease states, declines in impedance values at this frequency are most readily observe[35]. In the present study, it was found no correlations between the thickness or change percentage with the electrical properties of LPM in the patients with non-specific NSCLBP. It is consist with Rutkove's opinion[7]. He suggested that for ultrasound, energy is in the form of sound waves and the main interest is image reconstruction, but EIM holds the promise of serving as an indicator of disease status, thus being useful in clinical trials work and in monitoring effectiveness of treatment in individual patients. It concluded that EIM values and morphological parameter were independent factors for NSCLBP. EIM measurement might be more sensitive than ultrasound since the patients was young adults with moderate pain (average VAS value $5.19 \mathrm{~cm})$.

In all, the $R$ value increased, and no change of the $X$ value was in the NSCLBP group in the study. It indicates that the LPM was atrophic or the permeability of the cellular membranes in the patients with NSCLBP was changed but the integrity is as same as healthy individuals. We suggested that the content of extracellular and intracellular water changed the LPM geometry and fat infiltration were highly likely to be the cause and for young patients, the morphology of LPM have not been changed yet. Because Sions et al[36] reported older adults with LBP had a greater average multifidus muscle-to-fat index and smaller 
average erector spinae relative muscle CSA when compared to control participants without LBP. Mengiardi et al[37] using proton magnetic resonance spectroscopy reported significant multifidus intramuscular fat has been demonstrated in younger adults with NSCLBP when compared to healthy controls. Wan et al[38] applied MRI to assess patients with acute and chronic LBP and found there are special undergoing atrophy and fatty infiltration in the LPM. For further verifying the inference, it needs more studies. Using EIM detects NSCLBP patients could find some significant changes in the LPM different from healthy people. These findings suggest that localized bioimpedance analysis has the potential of playing a substantial role in the diagnostic and therapeutic evaluation of NSCLBP.

\section{Limitations}

These findings may be somewhat limited by the small sample size. Only young adults were measured on one body location, thus limiting the representation of the results explains for all population with NSCLBP. Because of the obstacle from the anatomical structure lumbar crest, we did not measure the EIM parameters at transverse direction so that the anisotropy ratios of each variable were not calculated. Since muscle anisotropy represents the degree of columnar order in the arrangement of the fibers[39], the arrangement of LPM fibers in NSCLBP was lack for assessment in the study.

\section{Conclusion}

The electrical properties of bilateral LPM in young adults with NSCLBP were different from heathy individuals. At $50 \mathrm{kHz}, 100 \mathrm{kHz}$ and $200 \mathrm{kHz}$ current frequencies, the bilateral LPM of NSCLBP patients showed increased resistance, decreased phase angle and no significant change in reactance compared with healthy subjects. The ultrasound parameters of LPM showed no significant difference between young patients with NSCLBP and without. EIM measurement might be more sensitive than rehabilitative ultrasound for NSCLBP in young adults.

\section{Abbreviations}

CLBP: chronic low back pain;CSA: cross-sectional area; EIM: electrical impedance myography; GLM: general liner model; HC: healthy controls; LBP: low back pain; LPM: lumbar paraspinal muscle; MVC: maximum volunteer contraction; NSCLBP: non-specific chronic low back pain; R: resistance; $X$ : reactance; PA: phase angle; ODI: Oswestry Disability Index; RDQ: Roland-Morris Disability Questionnaire.

\section{Declarations}

\section{Ethical approval}

The study was approved by the Human Subjects Ethics Subcommittee of the First Affiliated Hospital of Sun Yat-sen University (registration number: 2020425) and performed according to the Declaration of Helsinki. Every participant was only enrolled in the study after signing a written informed consent. 


\section{Consent for publication}

Every participant was only enrolled in the study after signing a written informed consent. They agreed offered the personal data if required. All participants have consent for publication. The authors can offer the institutional consent form if it was needed.

\section{Availability of data and materials}

The datasets used and analyzed during the current study and available from the corresponding authors on reasonable request.

\section{Competing interests}

We declare that none of the authors has any financial or non-financial competing interests.

\section{Funding}

This work was supported by Development Center For Medical Science \& Technology National Health Commission of the People's Republic of China, grant number: DCMST-NHC-2019-AHT-01.

\section{Authors' contributions}

Hongjiang Wang and Chuhuai Wang wrote the main manuscript text. Qiuhua Yu, Ziyan Fan and Jiaxuan Zheng collected the questionnaires, EIM data and ultrasound measurement. Le Li helped to analysis the data and design the study. Wai Leung Ambrose Lo and Hongjiang Wang prepared the figure and tables. All authors reviewed and approved the final manuscript.

\section{Acknowledgments}

The authors would like to sincerely thank the study's participants for their cooperation and support during the data collection: Liu Xin. Thank Li Tingni for helping to solve with data analysis problems.

\section{References}

1. Thiese, M.S., et al., Prevalence of low back pain by anatomic location and intensity in an occupational population. BMC Musculoskelet Disord, 2014. 15: p. 283.

2. Meucci, R.D., A.G. Fassa, and N.M. Faria, Prevalence of chronic low back pain: systematic review. Rev Saude Publica, 2015. 49.

3. Ma, K., et al., The Chinese Association for the Study of Pain (CASP): Consensus on the Assessment and Management of Chronic Nonspecific Low Back Pain. Pain Res Manag, 2019. 2019: p. 8957847. 
4. van Dieën, J.H., et al., Motor Control Changes in Low Back Pain: Divergence in Presentations and Mechanisms. Journal of Orthopaedic \& Sports Physical Therapy, 2019. 49(6): p. 370-379.

5. Panjabi, M.M., The stabilizing system of the spine. Part II. Neutral zone and instability hypothesis. J Spinal Disord, 1992. 5(4): p. 390-6; discussion 397.

6. van Dieen, J.H., J. Cholewicki, and A. Radebold, Trunk muscle recruitment patterns in patients with low back pain enhance the stability of the lumbar spine. Spine (Phila Pa 1976), 2003. 28(8): p. 83441.

7. Rutkove, S.B., Electrical impedance myography: Background, current state, and future directions. Muscle Nerve, 2009. 40(6): p. 936-46.

8. Roy, B., S.B. Rutkove, and R.J. Nowak, Electrical impedance myography as a biomarker of inclusion body myositis: A cross-sectional study. Clin Neurophysiol, 2020. 131(2): p. 368-371.

9. Leitner, M.L., et al., Electrical impedance myography for reducing sample size in Duchenne muscular dystrophy trials. Ann Clin Transl Neurol, 2020. 7(1): p. 4-14.

10. Alix, J.J.P., et al., Multi-dimensional electrical impedance myography of the tongue as a potential biomarker for amyotrophic lateral sclerosis. Clin Neurophysiol, 2020. 131(4): p. 799-808.

11. Hu, C., et al., Correlation Between Muscle Structures and Electrical Properties of the Tibialis Anterior in Subacute Stroke Survivors: A Pilot Study. Front Neurosci, 2019. 13: p. 1270.

12. Statland, J.M., et al., Electrical impedance myography in facioscapulohumeral muscular dystrophy. Muscle Nerve, 2016. 54(4): p. 696-701.

13. Stalberg, E., et al., Standards for quantification of EMG and neurography. Clin Neurophysiol, 2019. 130(9): p. 1688-1729.

14. Wang, Y., et al., Electrical Impedance Myography for Assessing Paraspinal Muscles of Patients with Low Back Pain. J Electr Bioimpedance, 2019. 10(1): p. 103-109.

15. Ching, C.T., et al., Characterization of the muscle electrical properties in low back pain patients by electrical impedance myography. PLoS One, 2013. 8(4): p. e61639.

16. Patrick, N., E. Emanski, and M.A. Knaub, Acute and chronic low back pain. Med Clin North Am, 2014. 98(4): p. 777-89, xii.

17. Lamoth, C.J.C., et al., Effects of chronic low back pain on trunk coordination and back muscle activity during walking: changes in motor control. European Spine Journal, 2005. 15(1): p. 23-40.

18. Schouppe, S., et al., Does experimentally induced pain-related fear influence central and peripheral movement preparation in healthy people and patients with low back pain? Pain, 2020. 161(6): 
p. $1212-1226$.

19. Chou, R., et al., Diagnosis and treatment of low back pain: A joint clinical practice guideline from the American college of physicians and the American pain society. Annals of Internal Medicine, 2007. 147(7): p. 478-491.

20. Chen, J., et al., Reliability and validity of the Chinese version of the behavioral pain scale in intubated and non-intubated critically ill patients: Two cross-sectional studies. Int J Nurs Stud, 2016. 61: p. 63-71.

21. Roland, M. and J. Fairbank, The Roland-Morris Disability Questionnaire and the Oswestry Disability Questionnaire. Spine, 2000. 25(24): p. 3115-3124.

22. Sweeney, N., C. O'Sullivan, and G. Kelly, Multifidus muscle size and percentage thickness changes among patients with unilateral chronic low back pain (CLBP) and healthy controls in prone and standing. Man Ther, 2014. 19(5): p. 433-9.

23. Kiesel, K.B., et al., Measurement of lumbar multifidus muscle contraction with rehabilitative ultrasound imaging. Man Ther, 2007. 12(2): p. 161-6.

24. Zhang, C., et al., Pain Catastrophizing Is Related to Static Postural Control Impairment in Patients with Nonspecific Chronic Low Back Pain: A Cross-Sectional Study. Pain Res Manag, 2020. 2020: p. 9629526.

25. Sanchez, B. and S.B. Rutkove, Electrical Impedance Myography and Its Applications in Neuromuscular Disorders. Neurotherapeutics, 2017. 14(1): p. 107-118.

26. Shiffman, C.A., H. Kashuri, and R. Aaron, Electrical impedance myography at frequencies up to 2 MHz. Physiol Meas, 2008. 29(6): p. S345-63.

27. Tarulli, A.W., et al., Electrical impedance myography in the assessment of disuse atrophy. Arch Phys Med Rehabil, 2009. 90(10): p. 1806-10.

28. De Lorenzo, A., et al., Predicting body cell mass with bioimpedance by using theoretical methods: a technological review. Journal of applied physiology (Bethesda, Md. : 1985), 1997. 82(5): p. 1542-58.

29. Coutinho, A.B.B., et al., Invasive electrical impedance myography at different levels of contraction of gastrocnemius muscle of rat. Rev Sci Instrum, 2020. 91(8): p. 084103.

30. Shiffman, C.A., et al., Resistivity and phase in localized BIA. Phys Med Biol, 1999. 44(10): p. 2409-29.

31. Li, X., et al., Electrical Impedance Myography for Evaluating Paretic Muscle Changes After Stroke. IEEE Trans Neural Syst Rehabil Eng, 2017. 25(11): p. 2113-2121. 
32. Wallwork, T.L., et al., The effect of chronic low back pain on size and contraction of the lumbar multifidus muscle. Man Ther, 2009. 14(5): p. 496-500.

33. Zhang, S., et al., Functional and Morphological Changes in the Deep Lumbar Multifidus Using Electromyography and Ultrasound. Sci Rep, 2018. 8(1): p. 6539.

34. Masse-Alarie, $\mathrm{H}$., et al., The side of chronic low back pain matters: evidence from the primary motor cortex excitability and the postural adjustments of multifidi muscles. Exp Brain Res, 2017. 235(3): p. 647-659.

35. Kortman, H.G., et al., Age-and gender-associated differences in electrical impedance values of skeletal muscle. Physiol Meas, 2013. 34(12): p. 1611-22.

36. Sions, J.M., et al., Trunk Muscle Characteristics of the Multifidi, Erector Spinae, Psoas, and Quadratus Lumborum in Older Adults With and Without Chronic Low Back Pain. J Orthop Sports Phys Ther, 2017. 47(3): p. 173-179.

37. Mengiardi, B., et al., Fat content of lumbar paraspinal muscles in patients with chronic low back pain and in asymptomatic volunteers: quantification with MR spectroscopy. Radiology, 2006. 240(3): p. 786-92.

38. Wan, Q., et al., MRI assessment of paraspinal muscles in patients with acute and chronic unilateral low back pain. Br J Radiol, 2015. 88(1053): p. 20140546.

39. Garmirian, L.P., A.B. Chin, and S.B. Rutkove, Discriminating neurogenic from myopathic disease via measurement of muscle anisotropy. Muscle Nerve, 2009. 39(1): p. 16-24.

\section{Figures}

a
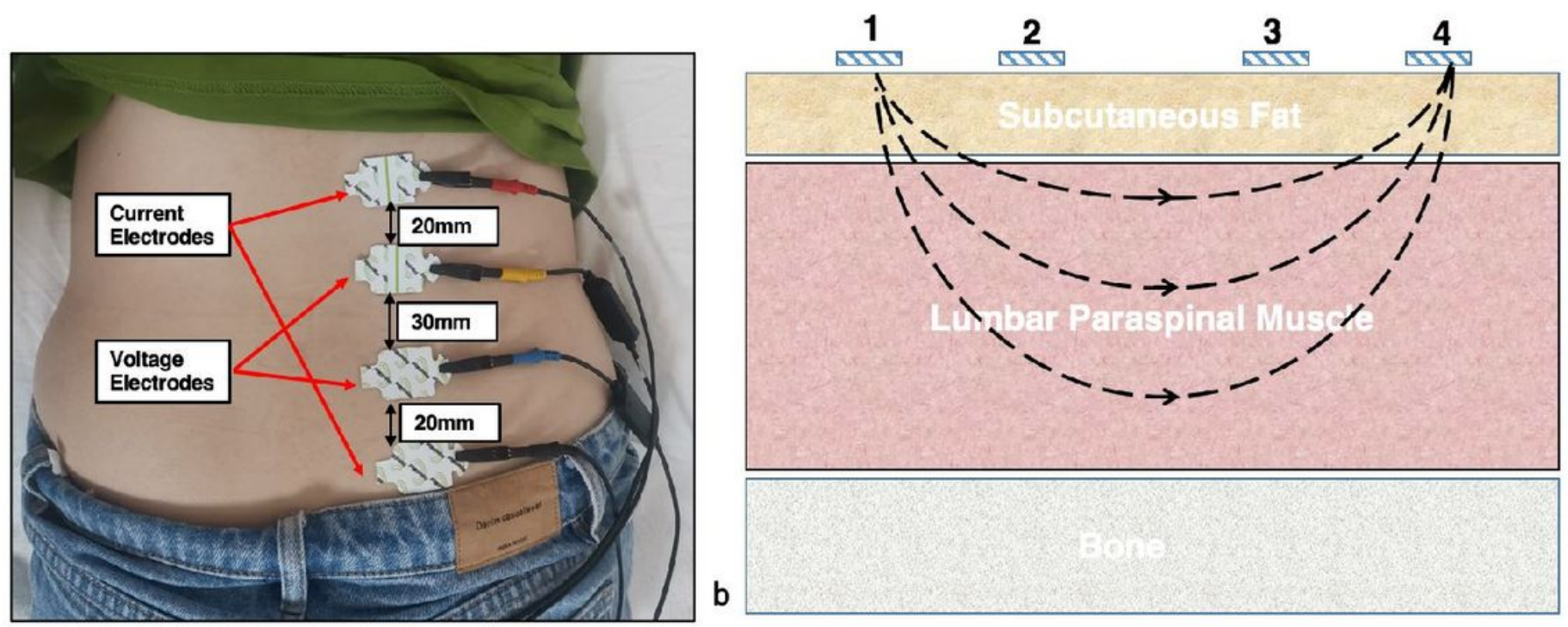


\section{Figure 1}

(a) One female subject was relaxed and in prone position on an instrumented bed. One pair of current electrodes (red and black) and one pair of voltage electrodes (yellow and blue) were linearly arranged along the surface of the right LPM on the muscle belly. (b) Demonstration of positions of EIM parameters. 1 and 4 were a pair of current electrodes of EIM. 2 and 3 were a pair of voltage electrodes of EIM. 